\title{
Prognostic implications of type 2 myocardial infarctions
}

\author{
Hazim El-Haddad, Elijah Robinson, Katrina Swett, Gretchen L. Wells* \\ Department of Internal Medicine-Section on Cardiology, Wake Forest School of Medicine, Winston-Salem, USA \\ Email: "'gwells@,wakehealth.edu
}

Received 26 July 2012; revised 30 August 2012; accepted 11 September 2012

\begin{abstract}
Purpose: A type 2 myocardial infarction (MI) is due to ischemia from increased oxygen demand or decreased supply. It is not an infrequent complication following non-cardiac surgery or in the setting of acute medical illness. The purpose of this study was to determine whether type 2 MIs had a worse prognosis than a type 1 MI. Methods: The hospital database of a large tertiary-level academic medical center was queried for all patients with a troponin $I \geq 1.6 \mathrm{ng} / \mathrm{mL}$ during a one-year period. The outcomes of patients with a type 2 MI were compared to those with a type 1 MI. Results: This retrospective study found that a type $2 \mathrm{MI}$ is more lethal than the usual type $1 \mathrm{MI}$, particularly among women and the elderly. There was no statistical difference in outcomes between those that occurred in the post-operative setting compared to those that occurred in the setting of acute medical illness. Conclusions: Type 2 MIs are common and are associated with increased mortality, as compared with type 1 MIs. To date, no guidelines exist for the management of this type of MI. Further research into underlying mechanisms resulting in type $2 \mathrm{MI}$ as well as potential treatment strategies is needed.
\end{abstract}

Keywords: Type 2 Myocardial Infarction; Postoperative Myocardial Infarction; Troponin; Sepsis

\section{INTRODUCTION}

Myocardial infarction (MI) is a major cause of death and disability worldwide. Cardiac troponin, a regulatory protein of the thin filament (actin) of cardiac muscle, is the standard biomarker for the diagnosis of acute MI [1]. Cardiac troponin (I or T) has near absolute specificity for myocardial tissue and high clinical sensitivity [2]. An increased value is defined as a measurement exceeding the 99th percentile of a normal reference population. Although elevation of troponin I or T reflects myocardial necrosis, it does not indicate its mechanism.

"Corresponding author.
Improvements in the diagnosis of MI led the World Health Organization to update the universal definition of MI. Currently there are five clinical classifications of MI as described by the Joint European Society of Cardiology (ESC)/American College of Cardiology (ACC)/American Heart Association (AHA)/World Heart Federation (WHF) Task Force for the Redefinition of Myocardial Infarction [3]:

Type 1 Spontaneous MI due to a primary cardiac event (e.g. plaque rupture);

Type 2 MI due to ischemia from increased oxygen demand or decreased supply;

Type 3 Sudden unexplained cardiac death;

Type 4a MI associated with percutaneous coronary intervention (PCI);

Type $4 \mathrm{~b}$ MI associated with stent thrombosis;

Type $5 \mathrm{MI}$ associated with coronary artery bypass grafting (CABG);

Early measurement of troponin in blood samples leads to early recognition of myocardial necrosis. In patients with type I myocardial infarction, prompt recognition and treatment have led to improvement in morbidity and mortality. Type $1 \mathrm{MI}$ is usually due to a spontaneous plaque rupture, although other rare causes exist (e.g., spontaneous coronary artery dissection or embolization of an epicardial vessel). Evidence-based treatment guidelines for type 1 myocardial infarction (both ST segment elevation and non-ST segment elevation) are available and updated periodically $[4,5]$.

Alternatively, type $2 \mathrm{MI}$ occurs from ischemia due to either increased oxygen demand or decreased supply (e.g., anemia, hypotension, tachycardia). The exact mechanism resulting in type $2 \mathrm{MI}$ is unknown. Increased troponin levels in type 2 MI are usually seen in the setting of severe medical illness (i.e., sepsis) or following non-cardiac surgery. Troponin elevations in both settings (medical illness and peri-operative period) are associated with increased morbidity and mortality. To date, no guidelines for the treatment of type 2 myocardial infarction exist. In order to determine the significance of type $2 \mathrm{MI}$, we compared the mortality of all individuals with a troponin $\mathrm{I} \geq 1.6 \mathrm{ng} / \mathrm{ml}$ during a 12 -month period at a large academic medical center. 


\section{METHODS}

\subsection{Study Population}

Following approval of the Wake Forest Baptist Medical Center Institutional Review Board, we examined the charts of all patients ages 18 years and older with a troponin $\mathrm{I} \geq 1.6 \mathrm{ng} / \mathrm{ml}$ hospitalized at an academic tertiary level medical center over a one-year period (January 1, 2009 to December 31, 2009). All cardiac troponin I assays were performed using fresh plasma within one hour following blood collection by standard venipuncture. All samples were analyzed on an Access Analyzer (Beckman Instruments, Beckman Coulter, Fullerton, CA, USA). At that time, laboratory values $\geq 1.6 \mathrm{ng} / \mathrm{ml}$ were considered abnormal. There were 1,012 medical records reviewed for which 807 individuals were included in the final analysis. Exclusion criteria were troponin elevations due to the following: sudden cardiac death, cardiac biomarker elevation following percutaneous intervention (PCI), stent thrombosis, post coronary artery bypass grafting or other cardiac surgery, chest trauma, treatment with chemotherapeutic drugs, myocarditis and Takotsubo's cardiomyopathy.

\subsection{Statistical Analysis}

Frequencies and percentages were calculated for categorical variables, and significance was assessed using chi-square tests. Means and standard deviations were calculated for age, and t test was used to assess signifycance. All analyses were performed using SAS 9.2 (Cary, $\mathrm{NC)}$.

\section{RESULTS}

We identified 807 individuals with a diagnosis of myocardial infarction over a one-year period (Table 1) of whom 512 (63.4\%) had type 1 MI and 295 (36.6\%) had type 2 MI. Of the patients with type $1 \mathrm{MI}, 237$ (46.3\%) had ST-segment and 275 (53.7\%) had non-ST-segment elevation MI. Of the patients with type 2 MI, 213 (72.2\%) occurred in the setting of an acute medical illness (Table 2), and $82(27.8 \%)$ occurred following non-cardiac surgery (Table 3). None of the ST-segment elevation MIs occurred in the postoperative setting or during an acute medical illness.

\subsection{Descriptive Statistics}

A greater number of men than women presented with myocardial infarction (529 men versus 278 women) (Table 4). There were also more Caucasians than African Americans (687 Caucasians versus 100 African Americans) and other populations (Table 4). The mean age for patients with ST-segment elevation MI was 62.4 (SD
13.8), non-ST-segment elevation MI was 64.1 and type 2 MI was 67.2 years. Of those who died, the mean age was 70.4 years, and of those who survived, the mean age was 63.8 years.

Table 1. Frequency of myocardial infarction: January 1, 2009December 31, 2009

\begin{tabular}{ccc}
\hline MI & Frequency & Percentage \\
\hline 1 (STEMI) & 237 & 29.4 \\
1 (NSTEMI) & 275 & 34.0 \\
2 & 295 & 36.6 \\
TOTAL & 807 & 100.0 \\
\hline
\end{tabular}

MI: Myocardial infarction; STEMI: ST-segment elevation myocardial infarction; NSTEMI: Non-ST-segment elevation myocardial infarction

Table 2. Type 2 myocardial infarction: Medical Illness.

\begin{tabular}{cc}
\hline Diagnosis & Number of patients \\
\hline Sepsis or sepsis shock & 55 \\
Hypertensive emergency & 32 \\
Gastrointestinal bleed & 31 \\
Respiratory failure (pneumonia; COPD) & 31 \\
Renal failure & 21 \\
Infection & 16 \\
Acute congestive heart failure & 10 \\
Supraventricular tachycardia & 9 \\
Pulmonary embolus & 8 \\
Total & $\mathbf{2 1 3}$ \\
\hline
\end{tabular}

Table 3. Type 2 myocardial infarction: Postoperative.

\begin{tabular}{cc}
\hline Type of procedure & Number of patients \\
\hline General surgery (cholecystectomy, & 17 \\
herniorrhaphy, bariatric) & 14 \\
Renal transplant & 13 \\
Orthopedic & \\
Vascular (lower extremity, abdominal & 8 \\
aortic aneurysm) & 7 \\
Oncology & 6 \\
Neurosurgery & 5 \\
Urology & 4 \\
Otolaryngology & 3 \\
Trauma & 2 \\
Ophthalmology & 1 \\
Burn & 1 \\
Total & 1 \\
Gynecology (total abdominal hysterectomy) & $\mathbf{8 2}$ \\
\hline
\end{tabular}


Table 4. Demographics of myocardial infarction January 1, 2009-December 31, 2009.

\begin{tabular}{llll}
\hline & STEMI & NSTEMI & Type 2 \\
\hline Gender & & & \\
Female $(\mathrm{n}=278)$ & $69(29.11 \%)$ & $83(30.18 \%)$ & $126(42.7 \%)$ \\
Male $(\mathrm{n}=529)$ & $168(70.89 \%)$ & $192(69.82 \%)$ & $169(57.29 \%)$ \\
Age & $62.4(30-97)$ & $64.1(22-90)$ & $67.2(21-99)$ \\
Race/Ethnicity & & & \\
$\begin{array}{l}\text { African American } \\
(\mathrm{n}=100)\end{array}$ & 15 & 28 & 57 \\
$\begin{array}{l}\text { Caucasian } \\
(\mathrm{n}=687)\end{array}$ & 210 & 244 & 233 \\
$\begin{array}{l}\text { Hispanic or } \\
\text { Latino }(\mathrm{n}=9)\end{array}$ & 3 & 1 & 5 \\
$\begin{array}{l}\text { Asian }(\mathrm{n}=1) \\
\text { Unknown }(\mathrm{n}=10)\end{array}$ & 8 & 0 & 0 \\
\hline
\end{tabular}

STEMI: ST-segment elevation myocardial infarction; NSTEMI: Non-STsegment elevation myocardial infarction; total number of patients $=807$.

\subsection{Mortality}

Patients with type 2 MIs were 6.9 times more likely to die than those with type 1 MIs $(\mathrm{p}<0.001)$. Of the 512 patients with type 1 MI, only 28 died. However, of 295 patients with type 2 MI, 84 died. Type 2 MIs occurring during a severe medical illness did not differ significantly from those type 2 MIs following non-cardiac surgery with respect to mortality (OR $0.75 ; 95 \%$, CI 0.42 - 1.34).

Gender was significantly associated with mortality $(\mathrm{p}=$ 0.002 ). Mortality rate in females was 1.9 times higher than that of males $(95 \%$, CI $1.25-2.81)$. However, the relationship between race and mortality was not significant $(\mathrm{p}=0.15)$.

\section{DISCUSSION}

We evaluated the frequency and consequences of both type 1 and 2 MIs over a one-year period and found that type 2 MIs are not infrequent and are associated with a higher mortality than type 1 MIs. In our series, patients with a type 2 MI were 6.9 times more likely to die than those with type 1 MI. The mortality among those patients with type 2 MI occurring during an acute medical illness was not significantly different from those occurring following non-cardiac surgery.

We found that more men and Caucasians suffered from type 1 [ST-segment elevation myocardial infarction (STEMI) and non-STEMI] as well as type 2 MI. The question of whether this finding reflects selection bias was not addressed in this study. The excess mortality observed in women, compared with men, in our study is consistent.

Type 2 MIs are due to myocardial oxygen supply demand imbalance. Ischemia occurs in patients with coro- nary artery disease during increased oxygen demand when inadequate flow increase occurs despite maximal vasodilatation of the coronary microvasculature. The most common cause of postoperative oxygen supply demand imbalance is tachycardia [6]. Studies have shown that heart rates $>80$ or $90 \mathrm{bpm}$ in patients with significant coronary artery disease whose preoperative resting heart rate is 50 to $60 \mathrm{bpm}$ can lead to prolonged ischemia and eventual infarction. Evidence for myocardial protection against perioperative myocardial ischemia by acute beta blockade was observed in a number of small studies $[7,8]$. Subsequent meta-analyses demonstrated conflicting results, suggesting either a reduction or no difference in cardiovascular events. For this reason, the American College of Cardiology/American Heart Association (ACC/ AHA) 2007 guidelines had administration of perioperative beta blockers as only a Class IIa recommend-dation [9]. Since that time, the largest randomized control trial investigating the effects of perioperative beta blockade, the Peri-Operative Ischemic Evaluation (POISE) Trial, found that beta blockade decreased the incidence of MI, but increased the incidence of death and stroke [10].

Other hemodynamic abnormalities associated with type 2 MIs include hypotension (associated with hypovolemia, anemia and systemic vasodilatation) and hypertension (associated with elevated stress hormones and vasoconstriction). Furthermore, in the setting of aggressive volume resuscitation, patients with underlying systolic and diastolic dysfunction may develop cardiac decompensation and type 2 MIs. However, hemodynamic instability and aggressive volume resuscitation were not reliable predictors of type $2 \mathrm{MI}$ in our series, leading to speculation that there could be other contributing mechanisms.

One small series demonstrated no coronary artery disease in 10 of 17 troponin-positive septic patients, in support of the hypothesis that other mechanisms may be responsible for the observed myocardial injury [11]. Both tumor necrosis factor alpha (TNF) and interleukin-1 (IL1) released in acute illness are known myocardial depressants [12], thus potentially resulting in the release of troponin. Microvascular dysfunction is well described in both sepsis and septic shock. Inflammatory mediators likely play a significant role in this setting by increasing the permeability of the endothelial monolayer for macromolecules resulting in troponin leakage [11].

Our study only addressed survival of acute hospitalization-not long-term mortality. Another series has demonstrated that post-operative MIs affect long-term survival [13]. One limitation of our retrospective study is that many patients with perioperative MIs do not experience symptoms of ischemia [14]. A prospective trial of all post-operative patients and acutely ill patients measuring cardiac biomarkers would address this question. 


\section{CONCLUSION}

Type 2 MIs are common in the setting of acute medical illness and following non-cardiac surgery, a frequent diagnosis now that highly-sensitive troponin assays are available. However, the exact mechanism of troponin elevation in this setting remains elusive. Studies have shown that even low-level elevations are prognostically significant. Our series demonstrated a significantly higher mortality in patients with type $2 \mathrm{MI}$ as compared to those with type $1 \mathrm{MI}$. Evidence-based guidelines for the treatment of type 1 MIs have likely led to marked improvement in survival. To date, however, no such guidelines exist for the treatment of type 2 MIs. Treatment strategies directed at potential underlying pathophysiology should be further investigated.

\section{ACKNOWLEDGEMENTS}

The authors gratefully acknowledge the administrative assistance of Amanda Burnette in the preparation of this manuscript.

\section{REFERENCES}

[1] Alpert, J.S., Thygesen, K., Antman, E. and Bassand, J.P. (2000) Myocardial infarction redefined-A consensus document of the Joint European Society of Cardiology/ American College of Cardiology Committee for the redefinition of myocardial infarction. Journal of the American College of Cardiology, 36, 959-969. doi:10.1016/S0735-1097(00)00804-4

[2] Jaffe, A.S., Ravkilde, J., Roberts, R., Naslund, U., Apple, F.S., Galvani, M. and Katus, H. (2000) It's time for a change to a troponin standard. Circulation, 102, 1216-1220. doi:10.1161/01.CIR.102.11.1216

[3] Thygesen, K., Alpert, J.S., White, H.D., Joint ESC/ACCF/ AHA/WHF Task Force for the Redefinition of Myocardial Infarction, Jaffe, A.S., Apple, F.S., Galvani, M., Katus, H.A., Newby, L.K., Ravkilde, J., Chaitman, B., Clemmensen, P.M., Dellborg, M., Hod, H., Porela, P., Underwood, R., Bax, J.J., Beller, G.A., Bonow, R., Van der Wall, E.E., Bassand, J.P., Wijins, W., Ferguson, T.B., Steg, P.G., Uretsky, B.F., Williams, D.O., Armstrong, P.W., Antman, E.M., Fox, K.A., Hamm, C.W., Ohman, E.M., Simoons, M.L., Poole-Wilson, P.A., Gurfinkel, E.P., Lopez-Sendon, J.L., Pais, P., Mendis, S., Zhu, J.R., Wallentin, L.C., Fernandez-Aviles, F., Fox, K.M., Parkhomenko, A.N., Priori, S.G., Tendera, M., Voipio-Pulkki, L.M., Vahanian, A., Camm, A.J., De Caterina, R., Dean, V., Dickstein, K., Filippatos, G., Funck-Brentano, C., Hellemans, I., Kristensen, S.D., McGregor, K., Sechtem, U., Silber, S., Tendera, M., Widimsky, P., Zamorano, J.L., Morais, J., Brener, S., Harrington, R., Morrow, D., Lim, M., Martinez-Rios, M.A., Steinhubl, S., Levine, G.N., Gibler, W.B., Goff, D., Tubaro, M., Dudek, D. and Al-Attar, N. (2012) Universal definition of myocardial infarction. Circulation, 116, 2634-2653.

[4] Antman, E.M., Hand, M., Armstrong, P.W., Bates, E.R., Green, L.A., Halasyamani, L.K., Hochman, J.S., Krum- holz, H.M., Lamas, G.A., Mullany, C.J., Pearle, D.L., Sloan, M.A., Smith, S.C.Jr., 2004 Writing Committee Members, Anbe, D.T., Kushner, F.G., Ornato, J.P., Jacobs, A.K., Adams, C.D., Anderson, J.L., Buller, C.E., Creager, M.A., Ettinger, S.M., Halperin, J.L., Hunt, S.A., Lytle, B.W., Nishimura, R., Page, R.L., Riegel, B., Tarkington, L.G. and Yancy, C.W. (2008) 2007 focused update of the ACC/AHA 2004 guidelines for the management of patients with ST-elevation myocardial infarction: A report of the American College of Cardiology/American heart association task force on practice guidelines developed in collaboration with the canadian cardiovascular society endorsed by the American academy of family physicians: 2007 writing group to review new evidence and update the ACC/AHA 2004 guidelines for the management of patients with ST-elevation myocardial infarction, writing on behalf of the 2004 writing committee. Circulation, 117, 296-329. doi:10.1161/CIRCULATIONAHA.107.188209

[5] Anderson, J.L., Adams, C.D., Antman, E.M., Bridges, C.R., Califf, R.M., Casey, D.E., Chavey, W.E.2., Fesmire, F.M., Hochman, J.S., Levin, T.N., Lincoff, A.M., Peterson, E.D., Theroux, P., Wenger, N.K., Wright, R.S., Smith, S.C.Jr., Jacobs, A.K., Adams, C.D., Anderson, J.L., Antman, E.M., Halperin, J.L., Hunt, S.A., Krumholz, H.M., Kushner, F.G., Lytle, B.W., Nishimura, R., Ornato, J.P., Page, R.L., Riegel, B. (2007) ACC/AHA 2007 guidelines for the management of patients with unstable angina/non-ST-elevation myocardial infarction: A report of the American College of Cardiology/American heart association task force on practice guidelines (Writing Committee to revise the 2002 Guidelines for the Management of Patients With Unstable Angina/Non-ST-elevation Myocardial Infarction) developed in collaboration with the American College of Emergency Physicians, the Society for Cardiovascular Angiography and Interventions, and the Society of Thoracic Surgeons endorsed by the American Association of Cardiovascular and Pulmonary Rehabilitation and the Society for Academic Emergency Medicine. Journal of the American College of Cardiology, 50, e1-e157. doi:10.1016/j.jacc.2007.02.013

[6] Landesberg, G., Beattie, W.S., Mosseri, M., Jaffe, A.S. and Alpert, J.S. (2009) Perioperative myocardial infarction. Circulation, 119, 2936-2944.

doi:10.1161/CIRCULATIONAHA.108.828228

[7] Auerbach, A.D. and Goldman, L. (2002) Beta-blockers and reduction of cardiac events in noncardiac surgery. JAMA, 287, 1435-1444. doi:10.1001/jama.287.11.1435

[8] Stevens, R.D., Burri, H. and Tramer, M.R. (2003) Pharmacologic myocardial protection in patients undergoing noncardiac surgery: A quantitative systematic review. Anesthesia \& Analgesia, 97, 623-633. doi:10.1213/01.ANE.0000074795.68061.16

[9] Fleisher, L.A., Beckman, J.A., Brown, K.A., Calkins, H., Chaikof, E.L., Fleischmann, K.E., Freeman, W.K., Froehlich, J.B., Kasper, E.K., Kersten, J.R., Riegel, B., Robb, J.F., Smith, S.C.Jr., Jacobs, A.K., Adams, C.D., Anderson, J.L., Antman, E.M., Buller, C.E., Creager, M.A., Ettinger, S.M., Faxon, D.P., Fuster, V., Halperin, J.L., Hiratzka, L.F., Hunt, S.A., Lytle, B.W., Nishimura, R., Ornato, J.P., Page, R.L., Riegel, B., Tarkington, L.G. and Yancy, C.W. 
(2007) ACC/AHA 2007 guidelines on perioperative cardiovascular evaluation and care for noncardiac surgery: A report of the American College of Cardiology/American Heart Association task force on practice guidelines (Writing committee to revise the 2002 guidelines on perioperative cardiovascular evaluation for noncardiac surgery) developed in collaboration with the American society of echocardiography, American society of nuclear cardiology, heart rhythm society, society of cardiovascular anesthesiologists, society for cardiovascular angiography and interventions, society for vascular medicine and biology, and society for vascular surgery. Journal of the American College of Cardiology, 50, e159-e242. doi:10.1016/j.jacc.2007.09.003

[10] POISE Study Group, Devereaux, P.J., Yang, H., Yusuf, S., Guyatt, G., Leslie, K., Villar, J.C., Xavier, D., Chrolavicius, S., Greenspan, L., Pogue, J., Pais, P., Liu, L., Xu, S., Malaga, G., Avezum, A., Chan, M., Montori, V.M., Jacka, M. and Choi, P. (2008) Effects of extended- release metoprolol succinate in patients undergoing noncardiac surgery (POISE trial): A randomised controlled trial. Lancet, 371, 1839-1847. doi:10.1016/S0140-6736(08)60601-7

[11] Ammann, P., Fehr, T., Minder, E.I., Gunter, C. and Bertel,
O. (2001) Elevation of troponin 1 in sepsis and septic shock. Intensive Care Medicine, 27, 965-969. doi:10.1007/s001340100920

[12] Kumar, A., Thota, V., Dee, L., Olson, J., Uretz, E. and Parrillo, J.E. (1996) Tumor necrosis factor and interleukin 1 beta are responsible for in vitro myocardial cell depression induced by human septic shock serum. The Journal of Experimental Medicine, 183, 949-958. doi:10.1084/jem.183.3.949

[13] McFalls, E.O., Ward, H.B., Santilli, S., Scheftel, M., Chesler, E. and Doliszny, K.M. (1998) The influence of perioperative myocardial infarction on long-term prognosis following elective vascular surgery. Chest, 113, 681- 686 . doi:10.1378/chest.113.3.681

[14] Devereaux, P.J., Xavier, D., Pogue, J., Guyatt, G., Sigamani, A., Garutti, I., Leslie, K., Rao-Melacini, P., Chrolavicius, S., Yang, H., MacDonald, C., Avezum, A., Lanthier, L., Hu, W. and Yusuf, S. on behalf of the POISE (PeriOperative ISchemic Evaluation) Investigators (2011) Characteristics and short-term prognosis of perioperative myocardial infarction in patients undergoing noncardiac surgery. Annals of Internal Medicine, 154, 523-528. 